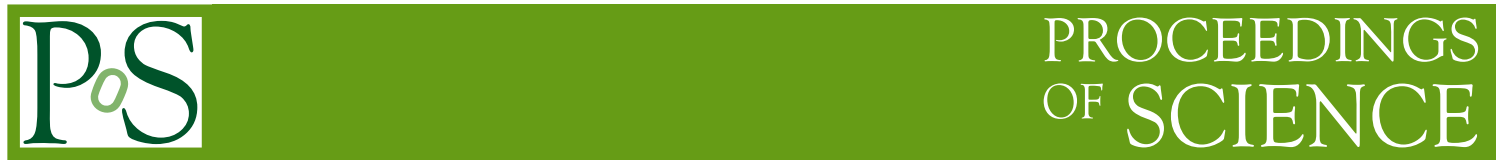

\title{
Lattice QCD with purely imaginary sources at zero and non-zero temperature.
}

\section{Massimo D’Elia}

Dipartimento di Fisica dell'Università di Pisa and INFN - Sezione di Pisa, Largo Pontecorvo 3, I-56127 Pisa, Italy

E-mail: delia@df.unipi.it

We discuss various aspects and recent progress concerning lattice QCD studies in the presence of external sources. We focus, in particular, on issues regarding QCD with non-zero imaginary chemical potentials or with a $\theta$-term, and on the properties of strongly interacting matter in the presence of electromagnetic background fields. 


\section{Introduction}

Exploring a quantum field theory in the presence of external sources is a common way to investigate its properties. A relevant example is the response to chemical potentials and background fields. We shall consider a class of sources which, in the Euclidean formulation, maintain the positivity of the path-integral formulation of the partition function, which for a non-Abelian lattice gauge theory is written as

$$
Z=\int \mathscr{D} U \mathscr{D} \psi \mathscr{D} \bar{\psi} e^{-\left(S_{G}[U]+\bar{\psi} M[U] \psi\right)}=\int \mathscr{D} U e^{-S_{G}[U]} \operatorname{det} M[U],
$$

so that their effect is directly explorable by numerical Monte-Carlo simulations, without the need to deal with a difficult sign problem.

In fact, such kind of sources are often considered in order to partially circumvent the sign problem which is met in the presence of real sources. The best known example is QCD in the presence of a baryon chemical potential $\mu_{B}$, whose partition function is written as

$$
Z\left(T, \mu_{B}\right)=\operatorname{Tr}\left(e^{-\frac{H_{\mathrm{QCD}}-\mu_{B} N_{B}}{T}}\right) .
$$

In the path-integral formulation a non-zero $\mu_{B}$ amounts to a modification of the quark action density $\bar{\psi}\left(m+\gamma_{v} D_{v}\right) \psi \rightarrow \bar{\psi}\left(m+\gamma_{v}\left(\partial_{v}+i\left(A_{v}-i \delta_{0 v} \mu\right)\right) \psi\right.$ where $\mu=\mu_{B} / 3$ is the quark chemical potential. If $\mu$ is real, the anti-hermiticity properties of $\gamma_{v} D_{v}$ are lost and the fermion determinant $\operatorname{det} M(\mu)$ becomes complex (sign problem), so that Monte-Carlo simulations cannot be used, unless opposite chemical potentials are given to up and down quarks (isospin chemical potential) or to left-handed and right-handed quarks (axial chemical potential $\mu_{5}$ ).

Approximate solutions consist in computing $\mu_{B}$ derivatives at $\mu_{B}=0$ (i.e. the cumulants of the baryon number distribution, or generalized susceptibilities), in order to reconstruct the free energy dependence as a Taylor expansion in $\mu_{B}$ [1], or in performing importance sampling by taking the positive part of the path-integral measure and then reweighting gauge configurations with the complex factor [2]. Both methods have significant limitations: reweighting in general fails as the statistics needed to correctly sample the partition function grow exponentially with the volume; Taylor expansion is limited to the small $\mu_{B}$ region, since the computational effort needed to determine the terms of the series grows very rapidly with the order of expansion.

An alternative is to introduce a purely imaginary quark chemical potential, $\mu=i \mu_{I}[3,4,5,6$, $7,8,9,10,11,12,13,14,15,16]$ : in this case the $\gamma_{5}$-hermiticity of the fermion matrix is preserved and the quark determinant is real and positive. In the standard way to introduce a chemical potential on the lattice [17], that amounts to adding a constant $U(1)$ phase to temporal links appearing in the Dirac operator, $U_{t}(n) \rightarrow e^{i a \mu_{I}} U_{t}(n)$, i.e. it is equivalent to a twist by an angle $\theta_{I}=\mu_{I} / T$ in the fermionic temporal boundary conditions. Charge conjugation implies that the partition function is even in $\mu$, so that switching to imaginary $\mu$ is actually like sending $\mu^{2} \rightarrow-\mu^{2}$, and analytic continuation can be performed in the $T-\mu^{2}$ plane, when possible, to infer properties at real $\mu$ from simulations at imaginary $\mu$.

The partition function at imaginary $\mu$ contains further interesting information. $Z\left(\theta_{I}\right)$ is of course periodic in $\theta_{I}$, with period $2 \pi$, but the additional symmetry of the path-integral measure and of the pure gauge term under center $Z_{N_{c}}$ transformations, where $N_{c}$ is the number of colors, implies 
that the partition function is actually $2 \pi / N_{c}$ periodic in $\theta_{I}$, i.e. $2 \pi$ periodic in $\theta_{B} \equiv \operatorname{Im}\left(\mu_{B}\right) / T$ [18]. Such a periodicity is directly linked to the fact that only globally colorless physical states (hence with an integer baryon number $n_{B}$ ) contribute to the partition function, both in the low $T$ and in the high $T$ phase, however it is differently realized, smoothly or with phase transitions at $\theta_{B}=\pi$ or odd multiples of it (known as Roberge-Weiss transitions [18]), depending on the effective degrees of freedom of the theory, which are hadrons below $T_{c}$ and quarks and gluons above. Lattice simulations have provided plenty of evidence of this different behavior from direct simulations at imaginary chemical potentials; moreover, the extraction of the Fourier coefficients of the free energy, considered as a periodic function of $\theta_{B}$, gives direct access to the canonical partition function, i.e. at fixed baryon number [19].

A closely analogous example is given by non-Abelian gauge theories, with or without dynamical fermions, with a topological $\theta$ term,

$$
Z(\theta)=\int \mathscr{D} U e^{-S_{G}[U]+i \theta Q[U]} \operatorname{det} M[U]
$$

where $Q=\int d^{4} x \frac{g_{0}^{2}}{64 \pi^{2}} \varepsilon_{\mu v \rho \sigma} G_{\mu \nu}^{a}(x) G_{\rho \sigma}^{a}(x)$ is the topological charge, which enters various aspects of $\mathrm{CP}$-violation in strong interactions. Also in this case a non-zero $\theta$ makes the path-integral measure complex, thus hindering direct Monte-Carlo simulations. One is usually interested in the $\theta$-dependence of various quantities of physical interest, including the vacuum energy and, at finite $T$, the free energy density, whose essential features can be reconstructed, in a Taylor expansion approach, in terms of the cumulants of the probability distribution of $Q$ at $\theta=0$. The introduction of a purely imaginary $\theta$ has been considered as an alternative since long [20], with many interesting results obtained in the last few years for QCD and QCD-like theories [21, 22, 23, 24, 25].

There is an interesting analogy between the physics at non-zero, real $\theta$ and that at non-zero, imaginary $\mu$, which is linked to the $2 \pi$ periodicity in $\theta$ deriving from the fact that $Q$ is integervalued. The analogy involves a sort of duality: the periodicity is non-trivially realized in the low $T$ phase of the theory, where the actual dependence is on $\theta / N_{c}$ and phase transitions appear at $\theta=\pi$ or odd multiples of it, while a smooth periodic behavior is realized in the deconfined phase. However, in this case the periodic behavior is on the same side where a sign problem occurs, so that its different realizations at high $T$ or low $T$ cannot be checked by direct simulations, as for imaginary $\mu$, but only indirectly, through the determination of the cumulants [26].

Electromagnetic sources have been considered since the early days of lattice simulations to probe the electromagnetic properties of hadrons [27]. An electromagnetic background field $a_{\mu}$ modifies the covariant derivative, for a quark with electric charge $q$, as follows:

$$
D_{\mu}=\partial_{\mu}+i g A_{\mu}^{a} T^{a} \rightarrow \partial_{\mu}+i g A_{\mu}^{a} T^{a}+i q a_{\mu} .
$$

The $\gamma_{5}$-hermiticity of the Dirac operator, hence the positivity of the quark determinant, is preserved only if $a_{\mu}$ is real valued: on the lattice, $S U(3)$ links appearing in the fermion matrix get multiplied by a pure phase factor, $U_{\mu}(n) \rightarrow U_{\mu}(n) u_{\mu}(n)$ where $u_{\mu}(n) \simeq \exp \left(i q a_{\mu}(n)\right) \in U(1)$, which is similar to adding an imaginary chemical potential. In the Euclidean path-integral formulation that corresponds to a non-zero imaginary electric field, while real electric fields lead to a sign problem. 
Numerical studies regarding the electric polarizabilities of hadrons usually involve such imaginary electric fields: the ground state energy shift, which at the lowest order is proportional to $|\mathbf{E}|^{2}$, is analitically continued from imaginary to real electric fields in order to determine the polarizability.

Magnetic fields, instead, are real, so that numerical simulations can be performed without any technical obstruction. That has opened the way to extensive simulations of QCD in the presence of magnetic background fields, whose interest is justified by the many phenomenological contexts where they may play a role, including the physics of compact astrophysical objects like magnetars [28], of non-central heavy ion collisions [29] and of the early Universe [30], which involve magnetic fields going from $10^{10}$ Tesla up to $10^{16}$ Tesla $\left(|e \mathbf{B}| \sim 1 \mathrm{GeV}^{2}\right)$.

Quantum Field Theories formulated in moving frames, e.g., Lorentz boosted or rotating, can also be viewed as systems with external sources, coupled respectively to the total momentum or to the angular momentum of the system. For instance, the partition function in a frame moving with speed $\mathbf{v}$ is written as

$$
Z(T, \mathbf{v})=\operatorname{Tr}\left(e^{-(H-\mathbf{v} \cdot \mathbf{P}) / T}\right)
$$

where $\mathbf{P}$ is the total momentum of the system. However, the path-integral representation of Eq. (1.5) leads to a sign problem, since the term coupled to $\mathbf{v}$ in the Euclidean action is purely imaginary, as can be easily verified even in the simplest case of the Euclidean path-integral for a non-relativistic particle after a Galileo transformation. Also in this case, the sign problem can be avoided by considering the speed $\mathbf{v}$ as a purely imaginary quantity, i.e. $\mathbf{v}=i \xi$, so that

$$
Z(T, \mathbf{v})=\operatorname{Tr}\left(e^{-(H-\mathbf{v} \cdot \mathbf{P}) / T}\right) \rightarrow \operatorname{Tr}\left(e^{-(H-i \xi \cdot \mathbf{P}) / T}\right)=\sum_{n}\left\langle n\left|e^{-(H-i \xi \cdot \mathbf{P}) / T}\right| n\right\rangle
$$

That, on its turn, can be viewed, for systems which are translationally invariant, as a modification of the standard thermodynamical trace, consisting in applying the translation operator $\exp (i \xi \cdot \mathbf{P}) / T$ to the right-hand side eigenstate $|n\rangle$. That has a representation in terms of a path-integral with spatially shifted, instead of standard periodic (or anti-periodic), boundary conditions in the time direction ( $\xi$ being the shift). Numerical simulations of such system are interesting, since the $\xi$-dependence of the theory can be used to derive the equation of state [31].

A similar continuation, from real to imaginary angular velocities, permits the numerical simulations of QCD in a rotating frame [32], which in this case can be useful and interesting by itself, because of the phenomenology related to heavy ion collisions and to compact stars.

In the following I will cover in more detail only some of the recent developments concerning the topics above. In cases where a sign problem exist, many efforts are being dedicated to the exploration of new approaches, which could eventually lead to an effective solution. Such efforts include Langevin simulations for generic complex actions [33], lattice simulations on a Lefschetz thimble [34], density of states methods [35], formulations in terms of dual variables [36], tensor Renormalization Group techniques [37], effective Polyakov loop models [38, 39] and many other approaches, which unfortunately will not be discussed in this context. 


\section{Imaginary chemical potentials}

The location of the (pseudo)critical temperature $T_{c}$ and the nature of the transition as a function of $\mu$ are two key issues for the QCD phase diagram. Determining $T_{c}(\mu)$ for small values of $\mu$ is a well defined goal for analytic continuation: $T_{c}$ is located for different values of $\mu_{I}$ and then it is fitted to a truncated Taylor series or to other expansions (e.g., Padé [40] approximants) and continued to real $\mu$. The reliability of the determination of the quadratic term, i.e. the curvature

$$
\frac{T_{c}\left(\mu_{B}\right)}{T_{c}}=1-\kappa\left(\frac{\mu_{B}}{T_{c}}\right)^{2}+O\left(\mu_{B}^{4}\right),
$$

has been verified in many different ways: by direct comparison with real $\mu$ simulations for theories without a sign problem [5] and by comparison with the outcome from other approaches, like reweighting [41] or Taylor expansion [42]. Determining non-linear contributions in $\mu^{2}$ is harder [5] and faces a typical problem for analytic continuation: contributions which are suppressed for $\mu^{2}<0$ may become important for $\mu^{2}>0$, because of the different signs in the Taylor expansion.

Recently, such studies have been extended to realistic discretizations of $N_{f}=2+1$ QCD with physical or almost physical quark masses in two different studies, the first adopting HISQ staggered quarks [12], the other adopting stout staggered quarks [15]. In both cases a tree level Symanzik improved gauge action and lattices with $N_{t}=6$ and $N_{t}=8$ have been adopted. Results for the curvature $\kappa$, Eq. (2.1), are reported in Fig. 1 and compared to previous determinations obtained by Taylor expansion: analytic continuation brings consistently larger results, with a discrepancy which is presently at the $2-\sigma$ level when systematic effects are taken into account [15]. A continuum extrapolation of these results is surely needed to clarify the issue, in view of a definite comparison with heavy ion phenomenology.

Like other methods to avoid the sign problem, analytic continuation will hardly say something definite regarding the possibile presence of a critical endpoint along the (pseudo)critical line, where the transition turns from crossover to first order. Numerical results show that the introduction of an imaginary $\mu$ tends to enlarge the region of small quark masses in the Columbia plot where the chiral transition is first order [4]; then, by analytic continuation (of a critical surface, in this case), a real $\mu$ would shrink the same region, thus disfavoring the appearance of a first order at the physical point. A safe conclusion, at present, is that the critical point, if any, is not to be found in the small $\mu$ region, and may not be directly connected to the chiral first order region taking place at $\mu=0$. That diminishes the chances to catch it before a complete solution to the sign problem is reached.

The phase structure at imaginary $\mu$ presents many interesting features by itself: the RobergeWeiss first order transition lines, which are present in the high- $T$ region and for $\theta_{B}=\operatorname{Im}\left(\mu_{B}\right) / T=$ $n \pi$, must stop with critical endpoints at some critical temperature $T_{R W}$, in order to match with the smooth periodic structure at low $T$. These are the only critical points in the QCD phase diagram whose existence can be predicted for sure (even if on the unphysical side); they also coincide, by exchange of the temporal direction with a spatial one, with the finite size transition at which charge conjugation symmetry breaks $[45,7]$. Recent studies, performed both with staggered $[7,8]$ and Wilson fermions $[9,10,11]$ for $N_{f}=2$ and $N_{f}=3$, have shown that such endpoints turn from first to second order, and then to first order again, as the quark masses are tuned from zero to infinity, 


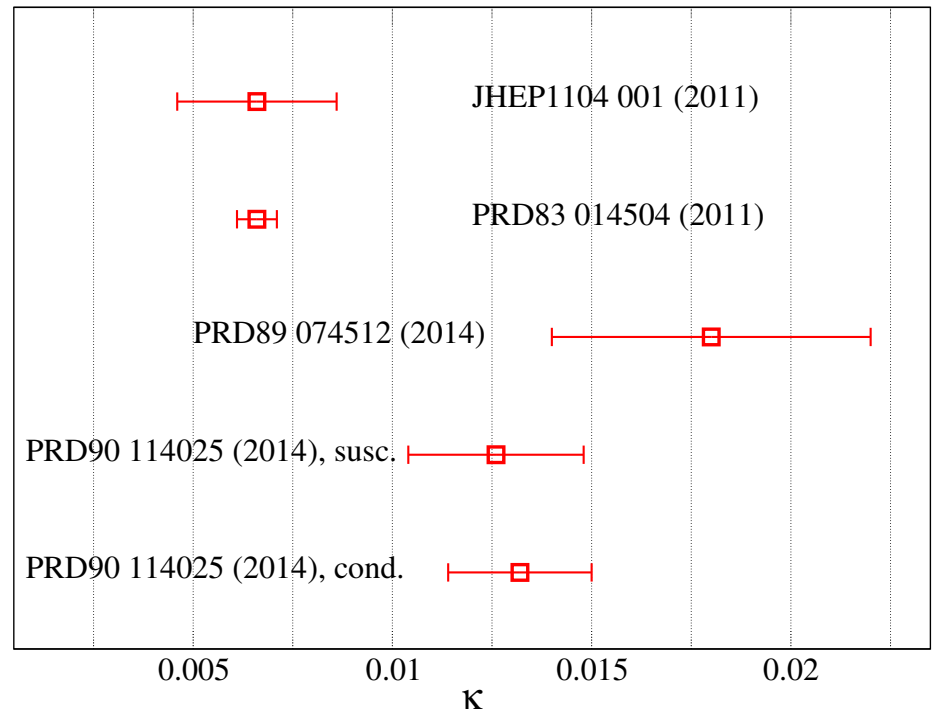

Figure 1: Curvature of the pseudo-critical line from different studies. From bottom to top: $i$ ) and ii) analytic continuation, chiral condensate and chiral susceptibility, stout staggered quarks [15]; iii) analytic continuation, chiral susceptibility with $\mu_{s}=\mu_{l}$, HISQ staggered quarks [12]; iv) Taylor expansion, chiral susceptibility, staggered quark p4-action [43]; $v$ ) Taylor expansion, chiral condensate, stout staggered quarks [44].

with tricritical points separating the different regions. The evolution of this phase structure can be monitored from $\operatorname{Im}\left(\mu_{B}\right) / T=\pi$ down to $\mu_{B}=0$, and the use of tricritical scaling [8] can be particularly helpful to extrapolate results to the chiral limit. That has permitted to make progress on a long-standing issue, like the order of the chiral transition for $N_{f}=2$ QCD in the massless limit [13]: the first order region which is present at $\operatorname{Im}\left(\mu_{B}\right) / T=\pi$ remains finite down to $\mu_{B}=0$, in agreement with previous lattice evidence [46]. Given the coarse lattices $\left(N_{t}=4\right.$ with standard staggered fermions), such a result is preliminary, but shows that the strategy is promising for future improved studies.

The non-trivial phase structure at imaginary $\mu$ has given rise to many model studies [47] and to further speculations about possible phenomena related to it [14]. An interesting aspect that we would like to stress is the possibility to test lattice methods, currently used to locate the critical endpoint (like looking at the radius of convergence of the Taylor series), on the critical points present at imaginary $\mu$, whose location is well known from direct simulations; this is something that should be done in the future, in order to better assess present systematic uncertainties on the determination of the critical point.

Simulations at imaginary $\mu$ provide useful information for many effective models used to explore the QCD phase diagram: in the same spirit of analytic continuation, the effective models are continued from real to imaginary chemical potentials, where the free parameters of the model are fixed against the results of lattice simulations. Examples are given by the effective Polyakov line action at finite chemical potential [38], or by studies where a Polyakov-loop-extended Nambu Jona-Lasinio (PNJL) model is directly matched to numerical results at imaginary $\mu$ [16]. Also various derivatives of the free energy with respect to the chemical potentials, i.e. susceptibilities 
and generalized susceptibilities, can be effectively computed by measuring the response of the thermal medium to non-zero imaginary sources, then exploiting analytic continuation. Systematic exploratory studies in this direction have been already performed [6]. In this context, one should also consider alternative ways for inserting the chemical potential on the lattice, see for instance Ref. [48], since that could change the effectiveness of analytic continuation.

\section{Electromagnetic background fields}

In the recent past the possibility of performing standard Monte-Carlo simulations, together with the interest related to various theoretical and phenomenological issues, has fostered many lattice investigations of the non-perturbative properties of strong interactions in the presence of a magnetic background field $\mathbf{B}$. While the initial primary interest, in connection with the phenomenology of non-central heavy ion collisions, was in the so-called Chiral Magnetic Effect (CME) [49], i.e. the electric charge separation taking place along $\mathbf{B}$ in the presence of local CP-violating fluctuations, various other topics have been explored, going from magnetic catalysis, i.e. the expected enhancement of chiral symmetry breaking induced by $\mathbf{B}$ [50], to the influence of magnetic fields on the QCD phase diagram and the magnetic properties of strongly interacting matter. Some studies have provided unexpected results, which have fostered further investigations, both at the level of lattice QCD simulations and at that of theoretical models.

Magnetic catalysis of the QCD vacuum, i.e. the increase of the chiral condensate as a function of $\mathbf{B}$, is a general phenomenon which has been confirmed by many investigations, adopting different discretizations and number of colors [51, 52, 53, 54, 55]. Instead, less expected developments have taken place at finite $T$. Early determinations $[56,53]$ of the dependence of the pseudo-critical temperature $T_{c}$ on $\mathbf{B}$, obtained by a standard staggered discretization with unphysical quark masses, showed a weak increasing behavior of $T_{c}$ with $\mathbf{B}$, which was also in agreement with most model predictions. However, later simulations, obtained with physical quark masses and closer to the continuum limit [57], have shown a decreasing behavior of $T_{c}$, which goes down by about $20 \%$ for $e B \sim 1 \mathrm{GeV}^{2}$; this is typically accompanied, in a region around $T_{c}$, by a decrease of the chiral condensate as a function of $B$, a phenomenon known as inverse magnetic catalysis. Some evidence for inverse magnetic catalysis has been obtained also by simulations of two-color QCD [55] and by adopting dynamical overlap fermions [58]. A similar behavior ( $T_{c}$ decreasing with $\left.B\right)$ was observed in the past in the case of chromo-magnetic backgrounds [59].

Regarding the chiral magnetic effect, lattice QCD studies have focused on various different aspects, like: $i$ ) looking directly at electric charge separation in a fixed topological and magnetic background [60]; ii) looking at the enhancement of longitudinal electric current fluctuations in a magnetic background [61]; iii) determining the longitudinal electric current in the presence of a magnetic background and an axial chemical potential [62]; iv) measuring the correlation between electric polarization and topological charge in the presence of a magnetic background [63]. In general, results show a suppression of the effect by about one order of magnitude with respect to model predictions (a similar suppression is observed also for the so-called axial magnetic effect [64]). However, lattice systematics, in particular regarding a correct discretization of chiral fermions, should be further checked in the future. 
Many efforts have been dedicated to investigate the magnetic response of strongly interacting matter, i.e. the dependence of its free energy on the magnetic background, which reveals whether the material is paramagnetic or diamagnetic. This is, in principle, a clear-cut question for lattice QCD simulations, since it regards the determination of purely equilibrium thermodynamical properties, contrary to other properties like the electrical conductivity of strongly interacting matter [65]. Nevertheless, obtaining a definite answer has been non-trivial since in lattice simulations, which usually adopts toroidal (hence compact) geometries in the spatial directions, the magnetic field is quantized, so that taking free energy derivatives with respect to $B$ is not a well defined operation. After some first attempts, aimed at determining only the spin component of the magnetization and suggesting diamagnetism at all temperatures [66], various improved methods have been devised. In particular one can: $i$ ) make use of thermodynamic integration in an extended parameter space in order to obtain directly free energy differences, instead of computing free energy derivatives $[67,68,69]$; ii) compute the magnetization in terms of pressure anisotropies in the direction parallel or orthogonal to $\mathbf{B}[70,71]$; iii) adopt an external field distribution which is not uniform and has zero net flux across the lattice torus, thus avoiding field quantization and allowing for a direct computation of derivatives [72]. Consistent results have been obtained by these methods and by different groups, showing that: i) strongly interacting matter is strongly paramagnetic above deconfinement $[67,68,69,71,72]$, with a response which is linear for magnetic fields going up to $e B \sim 0.1-0.2 \mathrm{GeV}^{2}[67,68]$ and a linear susceptibility which grows logaritmically with $T$ in the high- $T$ regime $[67,68,69]$; $i$ ) the magnetic activity is instead very weak below $T_{c}$; $i i i$ ) the QCD vacuum itself has a non-linear paramagnetic behavior [70]. A strong paramagnetic behavior in the deconfined phase is also the outcome of many model computations $[73,74]$.

In Fig. 2 we report a summary of the finite $T$ results, which include all studies performed at or close to the physical point (hence excluding early determinations with $N_{f}=2$ unimproved staggered quarks [67], which however provided qualitatively similar results), and are extrapolated to the continuum limit in two cases. The agreement is especially good when results from Refs. [68, $69,71]$ are compared: this is expected, since all three determinations adopt the same discretization of the theory (stout staggered quarks, while Ref. [72] adopts HISQ staggered quarks), hence the comparison is a nice test that different methods to determine the magnetic susceptibility provide consistent results. A few issues remain open, regarding in particular the computation of non-linear corrections to the free-energy dependence of $B$, which could give important contributions to the equation of state in the regime of large fields, relevant to the early stages of the Universe, and the clarification of the magnetic properties below $T_{c}$. Indeed, in a regime where the thermal medium can be well approximated by a purely pion gas, computations based on the Hadron Resonance Gas (HRG) model [74] lead to the prediction of weak diamagnetic behavior [68]: evidence for such a behavior at temperatures around $100 \mathrm{MeV}$ is still marginal [69], while it clearly emerges when an isospin chemical potential is added to the system, inducing pion condensation [75].

Lattice simulations have also provided substantial evidence for a direct influence of magnetic fields on the gluon sector, mediated by quark loop effects, which is expected due to the strongly interacting nature of QCD in the low energy regime. Large part of magnetic catalysis in the QCD vacuum is due to dynamical (sea) quark effects [52], which are also thought to be at the origin of inverse magnetic catalysis [76, 55]. Magnetic field induced modifications and anisotropies are also visible in observables like the gluon action density [53, 70] or the Polyakov loop [76]. Recently, 


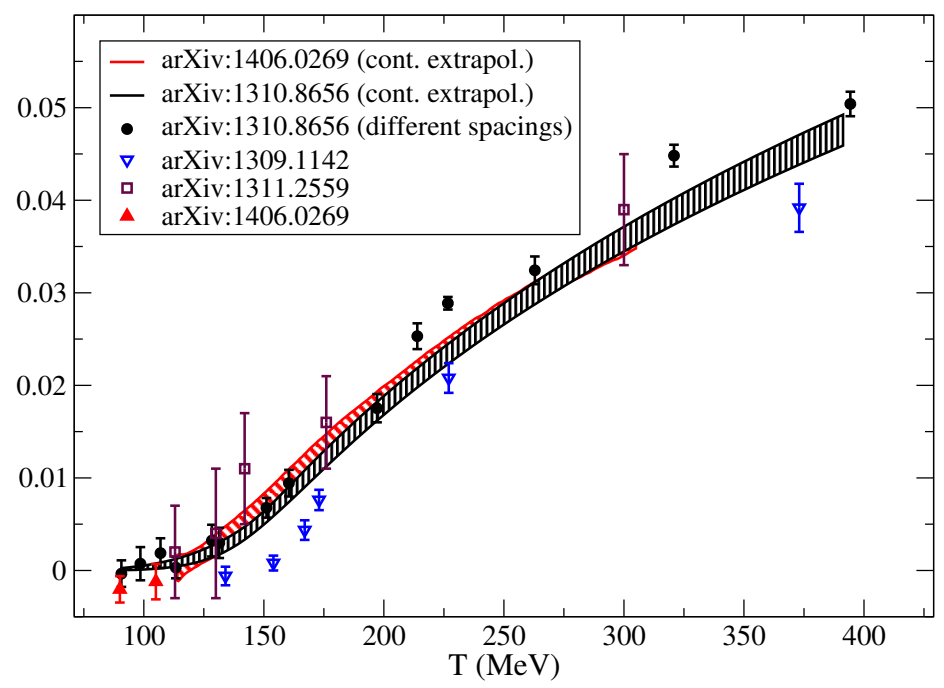

Figure 2: Various determinations of the magnetic susceptibility of strongly interacting matter as a function of $T$ from studies at or close to the physical point. Results from arXiv:1309.1142 [72] (HISQ staggered quarks) adopt a direct determination of the free energy derivatives in the presence of inhomogeneous magnetic fields; results from arXiv:1310.8656 [68] (stout staggered fermions) adopt thermodynamic integration, interpolating in $B$; results from arXiv:1311.2559 [71] (stout staggered quarks) have been obtained by determining the magnetization via pressure anisotropies; finally, results from arXiv:1406.0269 [69] (stout staggered quarks) adopt thermodynamic integration, interpolating in the quark condensate.

it has been found that even the static quark-antiquark potential undergoes significat modifications in the presence of a magnetic background [77]: the string tension becomes weaker/stronger in the direction parallel/orthogonal to $\mathbf{B}$, while the inverse occurs for the Coulomb coupling; such a behavior is consistent with some model computations [78].

Many studies have approached the problem of determining how the magnetic field influences the hadron spectrum. The interest has been driven mostly by the prediction for a superconductive phase of strong interactions above a critical magnetic field, which would be signalled by $\rho$ meson condensation [79]. Early numerical evidence about this phenomenon [80] has not been confirmed by later studies $[81,82]$, however the issue is still open. An open question is also that regarding the possible effects of the observed anisotropy of the static quark-antiquark potential on heavy meson states, and if these are eventually detectable in heavy ion experiments.

\section{QCD under combined external probes: topological effects in external fields}

In many situations of theoretical or phenomenological interest, one considers two different external sources at the same time, in order to probe new aspects of the theory. An illuminating example is furnished by the CME itself, where the combination of a magnetic background and of an effective local fluctuation of the $\theta$ parameter leads to the phenomenon of electric charge separation, to be eventually observed in heavy ion experiments.

Such combinations have been explored by lattice simulations in many contexts. Ref. [62] has explored the combined effect of an axial chemical potential and a magnetic background field in 
order to obtain a numerical estimate of the CME effect. Ref. [83] has considered a CP-violating combination of electric and magnetic backgrounds, i.e. such that $\mathbf{E} \cdot \mathbf{B} \neq 0$, in order to determine the induced effective $\theta$ term in the pure gauge sector, which is linked to the effective QED-QCD interactions in the pseudoscalar channel: in this case one relies on a double analytic continuation, since both $\mathbf{E}$ and the induced effective $\theta$ are purely imaginary. In Ref. [75], an isospin chemical potential has been introduced, together with a non-zero magnetic background, in order to explore the magnetic properties of isospin unbalanced matter. Various other possibilities could be explored along the same line: for instance, were it not for the presence of the sign problem, numerical simulation of dense, cold baryon matter in a magnetic background could open the exploration of interesting phenomena, like the De Haas - van Alphen effect, which could be relevant to heavy astrophysical objects.

A particularly interesting issue is the study of the combined application of a non-zero $\theta$-term and of an electric field. Indeed, a numerical non-perturbative determination of the dependence of the electric dipole moment (EDM) of the neutron on $\theta$, at least to the leading linear order, $d_{N}(\theta)=d_{N}^{(1)} \theta+O\left(\theta^{3}\right)$, would help putting an upper bound on $\theta$ based on the experimental upper bound on the EDM of the neutron. This is presently done by estimating $d_{N}^{(1)}$ by purely dimensional arguments. A possible way to determine $d_{N}(\theta)$ is to measure the three point function of an electromagnetic current between two neutron sources at non-zero $\theta .\left\langle N\left(\mathbf{p}_{1}, s_{1}\right)\left|V_{\mu}^{E M}\right| N\left(\mathbf{p}_{0}, s_{0}\right)\right\rangle_{\theta}$. The sign problem emerging from a non-zero $\theta$ can be approached by reweighting configurations sampled at $\theta=0$ [84]. An interesting alternative is to introduce an imaginary $\theta$ and then perform analytic continuation [23], the imaginary $\theta$ being introduced, after an anomalous axial transformation, as a $\gamma_{5}$ mass term in the fermionic action; a recent study [25] adopting this strategy has led to the upper bound $|\theta| \lesssim 7.6 \times 10^{-11}$. A different strategy, which has been proposed time ago [85], is to introduce an external electric background and measure the EDM from the energy difference between states with different spins in the presence of a $\theta$ term:

$$
\mathscr{E}_{+}^{\theta}\left(E_{z}\right)-\mathscr{E}_{-}^{\theta}\left(E_{z}\right)=d_{N}^{(1)} \theta E_{z}+O\left(\theta^{3}\right) .
$$

From a computational point of view, that could be very convenient, since it requires the determination of a two-point instead of a three-point function. However, one has to face two different sign problems at the same time, emerging from the non-zero electric field and from the non-zero $\theta$. A possibility could be to combine imaginary $\theta=i \theta_{I}$ and imaginary electric fields $\mathbf{E}=i \mathbf{E}_{I}$, perform direct determinations of the two point function

$$
\mathscr{E}_{+}^{\theta_{I}}\left(E_{I z}\right)-\mathscr{E}_{-}^{\theta_{I}}\left(E_{I z}\right) \simeq(i)^{2} d_{N}^{(1)} \theta_{I} E_{I z}=-d_{N}^{(1)} \theta_{I} E_{I z}
$$

and then rely on a double analytic continuation in the two different imaginary sources: preliminary results obtained by this approach have been reported in Ref. [22].

\section{Conclusions}

The insertion of external sources or background fields is a common way to probe the properties of Quantum Field Theories. In the context of lattice QCD simulations, one needs that the positivity of the path-integral measure be preserved by the insertion, otherwise standard Monte-Carlo methods cannot be used (sign problem). 
In the case of magnetic background fields, no such problem appears, so that lattice QCD simulations have been able to provide, in the last few years, plenty of information about the QCD phase diagram in a magnetic background and the magnetic properties of strongly interacting matter at zero and finite $T$. Peculiar phenomena have also been found, like inverse magnetic catalysis or magnetic field induced anisotropies in pure gauge quantities, which claim for further investigations in the next few years.

In other cases, which include quark chemical potentials, electric background fields and the $\theta$-term, the insertion makes the path-integral measure complex. A possible way out, in these cases, is to adopt purely imaginary sources, even if that does not correspond to the physical interesting case. One then typically relies on analytic continuation in order to extract results for the real case, however, the information obtained in the presence of imaginary sources can be interesting by itself or for direct comparison with other models and effective field theories.

One of the advantages of performing numerical simulations at non-zero external sources, with respect to the standard computation of expectation values at zero source in a Taylor expansion approach, is that one typically needs correlations functions and cumulants of lower order, with a great benefit in terms of noise and of computational effort. The trade-off is given by the fact that one has to perform dedicated simulations at non-zero sources, however there is still room for various improvements that should be explored in the future.

In this review we have discussed only a small part of the progress made in the field in the recent past. In particular, we could not cover the large efforts which are being made in order to perform direct simulations in the presence of sources which make the path-integral measure complex, i.e. in order to provide real, instead of imaginary, solutions to the sign problem.

\section{References}

[1] C. R. Allton et al., Phys. Rev. D 66, 074507 (2002); Phys. Rev. D 71, 054508 (2005); R. V. Gavai and S. Gupta, Phys. Rev. D 68, 034506 (2003); Phys. Rev. D 71, 114014 (2005).

[2] I. M. Barbour, S. E. Morrison, E. G. Klepfish, J. B. Kogut and M. P. Lombardo, Nucl. Phys. Proc. Suppl. 60A, 220 (1998); Z. Fodor, S. D. Katz, Phys. Lett. B 534 (2002) 87; JHEP 0203, 014 (2002).

[3] M.G. Alford, A. Kapustin, and F. Wilczek, Phys. Rev. D 59, 054502 (1999); M.-P. Lombardo, Nucl. Phys. Proc. Suppl. 83, 375 (2000); P. de Forcrand and O. Philipsen, Nucl. Phys. B 642, 290 (2002); Nucl. Phys. B 673, 170 (2003); M. D’Elia and M. P. Lombardo, Phys. Rev. D 67, 014505 (2003); Phys. Rev. D 70, 074509 (2004); H. S. Chen and X. Q. Luo, Phys. Rev. D 72, 034504 (2005); P. Giudice and A. Papa, Phys. Rev. D 69, 094509 (2004); V. Azcoiti, G. Di Carlo, A. Galante and V. Laliena, JHEP 0412, 010 (2004); Nucl. Phys. B 723, 77 (2005); Y. Shinno and H. Yoneyama, arXiv:0903.0922 [hep-lat]; F. Karbstein, M. Thies, Phys. Rev. D 75, 025003 (2007); L. K. Wu, X. Q. Luo and H. S. Chen, Phys. Rev. D 76, 034505 (2007); P. Cea, L. Cosmai, M. D'Elia and A. Papa, JHEP 0702, 066 (2007); S. Conradi and M. D’Elia, Phys. Rev. D 76, 074501 (2007); M. D’Elia, F. Di Renzo and M. P. Lombardo, Phys. Rev. D 76, 114509 (2007); K. Nagata and A. Nakamura, Phys. Rev. D 83, 114507 (2011).

[4] P. de Forcrand and O. Philipsen, JHEP 0701, 077 (2007); JHEP 0811, 012 (2008).

[5] P. Cea, L. Cosmai, M. D’Elia and A. Papa, Phys. Rev. D 77, 051501 (2008); Phys. Rev. D 81, 094502 (2010); P. Cea, L. Cosmai, M. D’Elia, C. Manneschi and A. Papa, Phys. Rev. D 80, 034501 (2009); P. Cea, L. Cosmai, M. D’Elia, A. Papa and F. Sanfilippo, Phys. Rev. D 85, 094512 (2012). 
[6] M. D'Elia and F. Sanfilippo, Phys. Rev. D 80, 014502 (2009); T. Takaishi, P. de Forcrand and A. Nakamura, PoS LAT 2009, 198 (2009).

[7] M. D'Elia and F. Sanfilippo, Phys. Rev. D 80, 111501 (2009); C. Bonati, G. Cossu, M. D'Elia and F. Sanfilippo, Phys. Rev. D 83, 054505 (2011).

[8] P. de Forcrand and O. Philipsen, Phys. Rev. Lett. 105, 152001 (2010).

[9] O. Philipsen and C. Pinke, Phys. Rev. D 89, 094504 (2014).

[10] A. Alexandru and A. Li, PoS LATTICE 2013, 208 (2013).

[11] L.-K. Wu and X.-F. Meng, Phys. Rev. D 87, 094508 (2013); Phys. Rev. D 90, no. 9, 094506 (2014).

[12] P. Cea, L. Cosmai and A. Papa, Phys. Rev. D 89, 074512 (2014).

[13] C. Bonati, P. de Forcrand, M. D’Elia, O. Philipsen and F. Sanfilippo, Phys. Rev. D 90, no. 7, 074030 (2014).

[14] K. Nagata, K. Kashiwa, A. Nakamura and S. M. Nishigaki, arXiv:1410.0783 [hep-lat].

[15] C. Bonati, M. D’Elia, M. Mariti, M. Mesiti, F. Negro and F. Sanfilippo, Phys. Rev. D 90, no. 11, 114025 (2014).

[16] J. Sugano, J. Takahashi, M. Ishii, H. Kouno and M. Yahiro, Phys. Rev. D 90, no. 3, 037901 (2014); J. Takahashi, H. Kouno and M. Yahiro, Phys. Rev. D 91, no. 1, 014501 (2015); J. Takahashi, J. Sugano, M. Ishii, H. Kouno and M. Yahiro arXiv:1410.8279 [hep-lat]; T. Makiyama et al., arXiv:1502.06191 [hep-lat].

[17] P. Hasenfratz and F. Karsch, Phys. Lett. B 125, 308 (1983); R. V. Gavai, Phys. Rev. D 32, 519 (1985).

[18] A. Roberge and N. Weiss, Nucl. Phys. B 275, 734 (1986).

[19] A. Hasenfratz and D. Toussaint, Nucl. Phys. B 371, 539 (1992); S. Kratochvila and P. de Forcrand, PoS LAT2005, 167 (2006); Nucl. Phys. Proc. Suppl. 153, 62 (2006); A. Alexandru, M. Faber, I. Horvath and K. F. Liu, Phys. Rev. D 72, 114513 (2005); S. Ejiri, Phys. Rev. D 78, 074507 (2008); C. Gattringer and L. Liptak, Phys. Lett. B 697, 85 (2011); A. Li, A. Alexandru, K. F. Liu and X. Meng, Phys. Rev. D 82, 054502 (2010); A. Alexandru and U. Wenger, Phys. Rev. D 83, 034502 (2011); A. Li, A. Alexandru and K. F. Liu, Phys. Rev. D 84, 071503 (2011); J. Danzer and C. Gattringer, Phys. Rev. D 86, 014502 (2012).

[20] G. Bhanot and F. David, Nucl. Phys. B 251, 127 (1985).

[21] V. Azcoiti, G. Di Carlo, A. Galante and V. Laliena, Phys. Rev. Lett. 89, 141601 (2002); M. Imachi, M. Kambayashi, Y. Shinno and H. Yoneyama, Prog. Theor. Phys. 116, 181 (2006); B. Alles and A. Papa, Phys. Rev. D 77, 056008 (2008); H. Panagopoulos and E. Vicari, JHEP 1111, 119 (2011).

[22] T. Izubuchi, S. Aoki, K. Hashimoto, Y. Nakamura, T. Sekido and G. Schierholz, PoS LAT 2007, 106 (2007).

[23] S. Aoki et al. arXiv:0808.1428 [hep-lat].

[24] M. D'Elia and F. Negro, Phys. Rev. Lett. 109, 072001 (2012); Phys. Rev. D 88, no. 3, 034503 (2013).

[25] F.-K. Guo et al., arXiv:1502.02295 [hep-lat].

[26] C. Bonati, M. D’Elia, H. Panagopoulos and E. Vicari, Phys. Rev. Lett. 110, no. 25, 252003 (2013).

[27] G. Martinelli, G. Parisi, R. Petronzio and F. Rapuano, Phys. Lett. B 116, 434 (1982); C. W. Bernard, T. Draper, K. Olynyk and M. Rushton, Phys. Rev. Lett. 49, 1076 (1982). 
Lattice QCD with purely imaginary sources at zero and non-zero temperature.

[28] R. C. Duncan and C. Thompson, Astrophys. J. 392, L9 (1992).

[29] V. Skokov, A. Y. Illarionov and V. Toneev, Int. J. Mod. Phys. A 24, 5925 (2009); V. Voronyuk et al, Phys. Rev. C 83, 054911 (2011); A. Bzdak and V. Skokov, Phys. Lett. B 710, 171 (2012); W. -T. Deng and X. -G. Huang, Phys. Rev. C 85, 044907 (2012); K. Tuchin, Adv. High Energy Phys. 2013, 490495 (2013).

[30] T. Vachaspati, Phys. Lett. B 265, 258 (1991); D. Grasso and H. R. Rubinstein, Phys. Rept. 348, 163 (2001).

[31] L. Giusti and H. B. Meyer, JHEP 1111, 087 (2011); JHEP 1111, 087 (2011); L. Giusti and M. Pepe, Phys. Rev. Lett. 113, 031601 (2014).

[32] A. Yamamoto and Y. Hirono, Phys. Rev. Lett. 111, 081601 (2013) [arXiv:1303.6292 [hep-lat]].

[33] F. Karsch and H. W. Wyld, Phys. Rev. Lett. 55, 2242 (1985); G. Aarts and I. O. Stamatescu, JHEP 0809, 018 (2008); G. Aarts, E. Seiler and I. O. Stamatescu, Phys. Rev. D 81, 054508 (2010); G. Aarts, E. Seiler, D. Sexty and I. O. Stamatescu, Phys. Rev. D 90, no. 11, 114505 (2014); G. Aarts, L. Bongiovanni, E. Seiler, D. Sexty and I. O. Stamatescu, Eur. Phys. J. A 49, 89 (2013); D. Sexty, arXiv:1410.8813 [hep-lat].

[34] M. Cristoforetti, F. Di Renzo, and L. Scorzato, Phys. Rev. D 86, 074506 (2012); M. Cristoforetti, F. Di Renzo, A. Mukherjee and L. Scorzato, Phys. Rev. D 88, no. 5, 051501 (2013); M. Cristoforetti $e$ al., Phys. Rev. D 89, no. 11, 114505 (2014).

[35] Z. Fodor, S. D. Katz and C. Schmidt, JHEP 0703, 121 (2007) [hep-lat/0701022]; K. Langfeld, B. Lucini and A. Rago, Phys. Rev. Lett. 109, 111601 (2012) [arXiv:1204.3243 [hep-lat]]; K. Langfeld and B. Lucini, Phys. Rev. D 90, no. 9, 094502 (2014) [arXiv:1404.7187 [hep-lat]]; S. Ejiri, Eur. Phys. J. A 49, 86 (2013) [arXiv:1306.0295 [hep-lat]]; J. Greensite, J. C. Myers and K. Splittorff, JHEP 1310, 192 (2013) [arXiv:1308.6712 [hep-lat]]; H. Saito, S. Ejiri, S. Aoki, K. Kanaya, Y. Nakagawa, H. Ohno, K. Okuno and T. Umeda, Phys. Rev. D 89, no. 3, 034507 (2014) [arXiv:1309.2445 [hep-lat]].

[36] C. Gattringer, PoS LATTICE 2013, 002 (2014). S. Chandrasekharan, Phys. Rev. D 82, 025007 (2010); J. Bloch, F. Bruckmann, N. Meyer and S. Schierenberg, JHEP 1208, 066 (2012); J. Bloch, Phys. Rev. D 86, 074505 (2012) C. Torrero, O. Borisenko, V. Kushnir, B. Allés and A. Papa, PoS LATTICE 2013, 338 (2014); T. Kloiber and C. Gattringer, arXiv:1410.3216 [hep-lat]; M. Kniely and C. Gattringer, arXiv:1502.00788 [hep-lat]; P. de Forcrand, J. Langelage, O. Philipsen and W. Unger, Phys. Rev. Lett. 113, no. 15, 152002 (2014) [arXiv:1406.4397 [hep-lat]];

[37] A. Denbleyker et al., Phys. Rev. D 89, no. 1, 016008 (2014); J. Unmuth-Yockey, Y. Meurice, J. Osborn and H. Zou, arXiv:1411.4213 [hep-lat].

[38] J. Greensite and K. Langfeld, Phys. Rev. D 90, no. 1, 014507 (2014); Phys. Rev. D 90, no. 11, 114507 (2014).

[39] J. Langelage, M. Neuman and O. Philipsen, JHEP 1409, 131 (2014).

[40] M. P. Lombardo, PoS LAT 2005, 168 (2006).

[41] Z. Fodor and S. D. Katz, arXiv:0908.3341 [hep-ph].

[42] E. Laermann, F. Meyer and M. P. Lombardo, J. Phys. Conf. Ser. 432, 012016 (2013); E. Laermann, F. Meyer and M. P. Lombardo, arXiv:1304.3247 [hep-lat].

[43] O. Kaczmarek, F. Karsch, E. Laermann, C. Miao, S. Mukherjee, P. Petreczky, C. Schmidt, W. Soeldner and W. Unger, Phys. Rev. D 83, 014504 (2011) [arXiv:1011.3130 [hep-lat]]. 
[44] G. Endrodi, Z. Fodor, S. D. Katz and K. K. Szabo, JHEP 1104, 001 (2011) [arXiv:1102.1356 [hep-lat]].

[45] T. DeGrand and R. Hoffmann, JHEP 0702, 022 (2007); B. Lucini, A. Patella and C. Pica, Phys. Rev. D 75, 121701 (2007).

[46] M. D’Elia, A. Di Giacomo and C. Pica, Phys. Rev. D 72, 114510 (2005); G. Cossu, M. D’Elia, A. Di Giacomo and C. Pica, arXiv:0706.4470 [hep-lat].

[47] H. Kouno, Y. Sakai, K. Kashiwa and M. Yahiro, J. Phys. G 36, 115010 (2009); Y. Sakai, K. Kashiwa, H. Kouno, M. Matsuzaki and M. Yahiro, Phys. Rev. D 79, 096001 (2009); J. Braun, L. M. Haas, F. Marhauser and J. M. Pawlowski, Phys. Rev. Lett. 106, 022002 (2011); Y. Sakai, T. Sasaki, H. Kouno and M. Yahiro, Phys. Rev. D 82, 076003 (2010); Phys. Rev. D 84, 091901 (2011); H. Kouno, M. Kishikawa, T. Sasaki, Y. Sakai and M. Yahiro, Phys. Rev. D 85, 016001 (2012); G. Aarts, S. P. Kumar and J. Rafferty, JHEP 1007, 056 (2010); J. Rafferty, JHEP 1109, 087 (2011); K. Morita, V. Skokov, B. Friman and K. Redlich, Phys. Rev. D 84, 076009 (2011); K. Kashiwa, T. Hell and W. Weise, Phys. Rev. D 84, 056010 (2011); V. Pagura, D. Gomez Dumm and N. N. Scoccola, Phys. Lett. B 707, 76 (2012); D. Scheffler, M. Buballa and J. Wambach, Acta Phys. Polon. Supp. 5, 971 (2012); K. Kashiwa and R. D. Pisarski, Phys. Rev. D 87, 096009 (2013); K. Kashiwa, T. Sasaki, H. Kouno and M. Yahiro, Phys. Rev. D 87, 016015 (2013); T. Misumi and T. Kanazawa, JHEP 1406, 181 (2014).

[48] R. V. Gavai and S. Sharma, Phys. Rev. D 85, 054508 (2012) [arXiv:1112.5428 [hep-lat]].

[49] A. Vilenkin, Phys. Rev. D 22, 3080 (1980); D. Kharzeev and A. Zhitnitsky, Nucl. Phys. A 797, 67 (2007); D. E. Kharzeev, L. D. McLerran and H. J. Warringa, Nucl. Phys. A 803, 227 (2008); K. Fukushima, D. E. Kharzeev and H. J. Warringa, Phys. Rev. D 78, 074033 (2008).

[50] A. Salam and J. A. Strathdee, Nucl. Phys. B 90, 203 (1975); H. Suganuma and T. Tatsumi, Annals Phys. 208, 470 (1991); S. Schramm, B. Muller, A. J. Schramm, Mod. Phys. Lett. A7, 973-982 (1992); S. P. Klevansky and R. H. Lemmer, Phys. Rev. D 39, 3478 (1989); K. G. Klimenko, Z. Phys. C 54, 323 (1992); Theor. Math. Phys. 94, 393 (1993); V. P. Gusynin, V. A. Miransky and I. A. Shovkovy, Phys. Rev. Lett. 73, 3499 (1994) [Erratum-ibid. 76, 1005 (1996)]; Nucl. Phys. B 462, 249 (1996); Phys. Lett. B 349, 477 (1995).

[51] P. V. Buividovich, M. N. Chernodub, E. V. Luschevskaya and M. I. Polikarpov, Phys. Lett. B 682, 484 (2010).

[52] M. D'Elia and F. Negro, Phys. Rev. D 83, 114028 (2011).

[53] E.-M. Ilgenfritz, M. Kalinowski, M. Muller-Preussker, B. Petersson and A. Schreiber, Phys. Rev. D $\mathbf{8 5}, 114504$ (2012).

[54] G. S. Bali, F. Bruckmann, G. Endrodi, Z. Fodor, S. D. Katz and A. Schafer, Phys. Rev. D 86, 071502 (2012).

[55] E.-M. Ilgenfritz, M. Muller-Preussker, B. Petersson and A. Schreiber, Phys. Rev. D 89, no. 5, 054512 (2014).

[56] M. D’Elia, S. Mukherjee and F. Sanfilippo, Phys. Rev. D 82, 051501 (2010).

[57] G. S. Bali et al., JHEP 1202, 044 (2012).

[58] V. G. Bornyakov, P. V. Buividovich, N. Cundy, O. A. Kochetkov and A. Schäfer, Phys. Rev. D 90, no. 3, 034501 (2014). 
Lattice QCD with purely imaginary sources at zero and non-zero temperature.

[59] P. Cea and L. Cosmai, JHEP 0302, 031 (2003); JHEP 0508, 079 (2005); P. Cea, L. Cosmai and M. D’Elia, JHEP 0712, 097 (2007).

[60] M. Abramczyk, T. Blum, G. Petropoulos and R. Zhou, PoS LAT 2009, 181 (2009).

[61] P. V. Buividovich, M. N. Chernodub, E. V. Luschevskaya and M. I. Polikarpov, Phys. Rev. D 80, 054503 (2009).

[62] A. Yamamoto, Phys. Rev. Lett. 107, 031601 (2011).

[63] G. S. Bali, F. Bruckmann, G. Endrödi, Z. Fodor, S. D. Katz and A. Schäfer, JHEP 1404, 129 (2014).

[64] V. Braguta, M. N. Chernodub, V. A. Goy, K. Landsteiner, A. V. Molochkov and M. I. Polikarpov, Phys. Rev. D 89, no. 7, 074510 (2014).

[65] A. Amato, G. Aarts, C. Allton, P. Giudice, S. Hands and J. I. Skullerud, Phys. Rev. Lett. 111, no. 17, 172001 (2013).

[66] G. S. Bali et al., Phys. Rev. D 86, 094512 (2012) [arXiv:1209.6015 [hep-lat]].

[67] C. Bonati, M. D’Elia, M. Mariti, F. Negro and F. Sanfilippo, Phys. Rev. Lett. 111, 182001 (2013).

[68] C. Bonati, M. D’Elia, M. Mariti, F. Negro and F. Sanfilippo, Phys. Rev. D 89, no. 5, 054506 (2014).

[69] G. S. Bali, F. Bruckmann, G. Endrödi, S. D. Katz and A. Schäfer, JHEP 1408, 177 (2014).

[70] G. S. Bali, F. Bruckmann, G. Endrodi, F. Gruber and A. Schaefer, JHEP 1304, 130 (2013).

[71] G. S. Bali, F. Bruckmann, G. Endrodi and A. Schafer, Phys. Rev. Lett. 112, 042301 (2014).

[72] L. Levkova and C. DeTar, Phys. Rev. Lett. 112, no. 1, 012002 (2014).

[73] M. M. Anber and M. Ünsal, JHEP 1412, 107 (2014); V. D. Orlovsky and Y. A. Simonov, Phys. Rev. D 89, no. 5, 054012 (2014); arXiv:1406.1056 [hep-ph]; A. N. Tawfik and N. Magdy, Phys. Rev. C 90, no. 1, 015204 (2014); K. Kamikado and T. Kanazawa, JHEP 1501, 129 (2015).

[74] G. Endrödi, JHEP 1304, 023 (2013).

[75] G. Endrödi, Phys. Rev. D 90, no. 9, 094501 (2014).

[76] F. Bruckmann, G. Endrodi and T. G. Kovacs, JHEP 1304, 112 (2013).

[77] C. Bonati, M. D’Elia, M. Mariti, M. Mesiti, F. Negro and F. Sanfilippo, Phys. Rev. D 89, no. 11, 114502 (2014).

[78] V. A. Miransky and I. A. Shovkovy, Phys. Rev. D 66, 045006 (2002); E. J. Ferrer, V. de la Incera and X. J. Wen, arXiv:1407.3503 [nucl-th]; R. Rougemont, R. Critelli and J. Noronha, Phys. Rev. D 91, no. 6, 066001 (2015).

[79] M. N. Chernodub, Phys. Rev. Lett. 106, 142003 (2011).

[80] V. V. Braguta, P. V. Buividovich, M. N. Chernodub, A. Y. Kotov and M. I. Polikarpov, Phys. Lett. B 718, 667 (2012).

[81] Y. Hidaka and A. Yamamoto, Phys. Rev. D 87, no. 9, 094502 (2013).

[82] E. V. Luschevskaya and O. V. Larina, Nucl. Phys. B 884, 1 (2014); E. V. Luschevskaya, O. V. Teryaev and O. A. Kochetkov, arXiv:1411.4284 [hep-lat]; E. V. Luschevskaya, O. A. Kochetkov, O. V. Larina and O. V. Teryaev, arXiv:1411.0730 [hep-lat].

[83] M. D’Elia, M. Mariti and F. Negro, Phys. Rev. Lett. 110, no. 8, 082002 (2013).

[84] E. Shintani et al., Phys. Rev. D 72, 014504 (2005).

[85] E. Shintani et al., Phys. Rev. D 75, 034507 (2007). 